\title{
A Network of Epigenetic Regulators Guide Developmental Hematopoiesis In Vivo
}

\author{
Hsuan-Ting Huang $1,2,{ }^{,}$, Katie L. Kathrein ${ }^{2,}{ }^{,}$, Abby Barton ${ }^{2}$, Zachary Gitlin ${ }^{2}$, Yue-Hua \\ Huang $^{2}$, Thomas P. Ward ${ }^{2}$, Oliver Hofmann ${ }^{3}$, Anthony Dibiase ${ }^{2}$, Anhua Song $^{2}$, Svitlana \\ Tyekucheva $^{4,5}$, Winston Hide ${ }^{3,4}$, Yi Zhou ${ }^{2}$, and Leonard I. Zon ${ }^{1,2,3,6}$ \\ ${ }^{1}$ Department of Genetics, Harvard Medical School, Boston, MA 02115 \\ ${ }^{2}$ Stem Cell Program and Division of Pediatric Hematology/Oncology, Children's Hospital Boston \\ and Dana-Farber Cancer Institute, Howard Hughes Medical Institute, Boston, MA 02115 \\ ${ }^{3}$ Harvard Stem Cell Institute, Cambridge, MA 02138 \\ ${ }^{4}$ Department of Biostatistics, Harvard School of Public Health, Boston, MA 02115 \\ ${ }^{5}$ Department of Biostatistics and Computational Biology, Dana-Farber Cancer Institute, Boston, \\ MA 02115
}

\section{Abstract}

The initiation of cellular programs is orchestrated by key transcription factors and chromatin regulators that activate or inhibit target gene expression. To generate a compendium of chromatin factors that establish the epigenetic code during developmental hematopoiesis, a large-scale reverse genetic screen was conducted targeting orthologs of 425 human chromatin factors in zebrafish. A set of chromatin regulators was identified that target different stages of primitive and definitive blood formation, including factors not previously implicated in hematopoiesis. We identified 15 factors that regulate development of primitive erythroid progenitors and 29 factors that regulate development of definitive stem and progenitor cells. These chromatin factors are associated with SWI/SNF and ISWI chromatin remodeling, SET1 methyltransferase, CBP/P300/ HBO1/NuA4 acetyltransferase, HDAC/NuRD deacetylase, and Polycomb repressive complexes. Our work provides a comprehensive view of how specific chromatin factors and their associated complexes play a major role in the establishment of hematopoietic cells in vivo.

The programs of gene expression required for maintenance and differentiation of cell types are tightly regulated by a network of transcription factors and associated chromatin modifying factors to facilitate or suppress gene expression. Epigenetic information consists

\footnotetext{
Users may view, print, copy, download and text and data- mine the content in such documents, for the purposes of academic research, subject always to the full Conditions of use: http://www.nature.com/authors/editorial_policies/license.html\#terms

${ }^{6}$ Corresponding author: 300 Longwood Ave., Karp 07211, Children's Hospital Boston, Boston, MA 02115, Ph: 617-919-2069, Fax: 617-730-0222, zon@enders.tch.harvard.edu.

*These authors contributed equally to this work

Author Contributions: H.T.H. and K.L.K. performed all experiments and data analysis. A.B. and Z.G. assisted with morpholino microinjection, WISH, and data collection. Y-H.H. assisted with morpholino microinjection. T.P.W., Y.Z., A.S., and A.D. developed the screen database. Y.Z. initiated and assisted with bioinformatic analysis of chromatin factors. O.H. and W.H. generated the protein interaction network. S.T. performed the distribution analysis for the ChIP-seq data. H.T.H. and L.I.Z. conceived the study.
} 
of chemical modifications to both cytosine bases in DNA and histone proteins that fold the DNA into nucleosomes, as well as the repositioning, dissociation, and/or reconstitution of entire nucleosomes ${ }^{1}$. Mouse knockout models and zebrafish mutants have been used to investigate the role of chromatin factors in vertebrate development, but the majority of chromatin factors have yet to be characterized ${ }^{2-5}$.

Hematopoiesis is guided by cell-specific transcriptional regulators and associated chromatin factors that function to establish all mature blood cells ${ }^{6}$. This process is hallmarked by the establishment of hematopoietic stem cells (HSCs) and depends on the function of transcriptional regulators, Runx1, Scl, Lmo2, and chromatin factors, Mll and Bmi1, for stem cell production, self-renewal, and survival. Additional factors coordinate the specialization of HSCs into multilineage progenitors that generate differentiated cells of the peripheral blood lineages ${ }^{6}$. Members of the Polycomb (PcG) family have previously been identified as regulators of hematopoiesis. Bmil functions to positively regulate HSC proliferation by limiting cell cycle regulator expression, and HSCs with Bmil deficiency show impaired self-renewal capacity ${ }^{7}$. The Mi-2/NuRD complex regulates a set of HSC specific genes that maintain the HSC pool in the bone marrow. De-repression of these genes in Mi-2beta deficient HSCs exhausts the $\mathrm{HSC} \mathrm{pool}^{8}$. Several chromatin factors have been identified as leukemic translocation partners, underscoring the importance they have in normal development. $M L L$ is rearranged in the majority of infant leukemias with patients generally having poor clinical outcomes ${ }^{9}$. Similarly, translocations of PRDM16, another SET family member, is associated with a poor prognosis ${ }^{10}$. Both promote the development of normal HSCs and leukemic stem cells 9,11 .

To identify chromatin factors that function during developmental hematopoiesis, we have undertaken a large-scale in vivo reverse genetic morpholino-based screen targeting zebrafish orthologs of 425 human chromatin factors. The zebrafish provides a suitable platform for rapid screening to assay the function of chromatin factors in hematopoiesis due to their high fecundity, rapid development, evolutionary conservation, and ease in generating genetic knockdowns. We have identified 44 factors that affect the development of primitive and definitive blood, including 28 factors that have yet to be associated with hematopoiesis. We have also characterized different developmental stages during which these factors function, from the induction of stem and progenitor cells to differentiation into erythroid cells. By incorporating protein interaction data, we predict the BAF/PBAF, ISWI, SET1, CBP/P300/ $\mathrm{HBO} 1 / \mathrm{NuA} 4, \mathrm{HDAC} / \mathrm{NuRD}$, and PRC1/PRC2 complexes as required for blood development. Taken together, our screen provides a valuable resource for elucidating the in vivo network of chromatin regulators of hematopoietic development.

\section{Results}

\section{A screen for chromatin regulators of developmental hematopoiesis}

To identify the chromatin remodeling factors that function in developmental hematopoiesis, we conducted a large-scale in vivo reverse genetic screen targeting chromatin factors (Fig. 1a). We designed antisense oligonucleotide morpholinos to knock down expression of 488 zebrafish orthologs of 425 human chromatin factors (Supplementary Table 1). Our gene list included most of the known human factors containing chromatin binding, modifying, or 
remodeling domains curated from several public databases: CREMOFAC, SMART domain by NRDB, CDD at NCBI, Pfam, and ChromDB ${ }^{12-16} .488$ orthologous genes in zebrafish were identified by a reciprocal BLAST search of the unique human protein sequences into the zebrafish genome. Only 26 human proteins lacked a zebrafish ortholog.

Morpholinos targeting each chromatin factor were injected into single cell embryos at three concentrations. These doses typically give a range of phenotypes from a hypomorph to a near complete knockdown for most mRNA products, similar to an allelic series. In some cases, complete knockdown could not be achieved because of lower targeting efficiency or embryonic lethality. Post-injection, embryos were collected at specific timepoints, using both standard morphological features of the whole embryo and hours post-fertilization (hpf) to stage to minimize differences in embryonic development caused by the morpholino injection ${ }^{17}$. The embryos were then assayed for hematopoietic defects by whole-mount in situ hybridization (WISH). We conducted two screens simultaneously for primitive and definitive blood formation. For the primitive screen, developing erythrocytes in the posterior mesoderm of the embryo were assayed by $\beta$-globin e 3 expression at the 16 somite stage (ss), or $17 \mathrm{hpf}$ (Fig. 1b) ${ }^{18}$. For the definitive screen, the establishment of hematopoietic stem and progenitor cells (HSPCs) in the aorta, gonad, mesonephros region (AGM) was detected with $c-m y b$ and runxl expression at 36 hpf (Fig. 1c) ${ }^{19}$.

To establish the level of morpholino efficacy, the 21 splice blocking morpholinos targeting the chromodomain (CHD) gene family were assayed for splicing activity by reversetranscription polymerase chain reaction (RT-PCR). Of these 21 morpholinos, $10 \mathrm{did}$ not result in any hematopoietic defect. For the 10, one gene could not be evaluated because no PCR product was detected. Only one of the nine remaining morpholinos did not show altered splicing activity, resulting in an estimated false negative rate (FNR) of $11 \%$ for the screen (Supplementary Fig. 1a-b). To expand on this limited approach, we verified the splicing activity of an additional 48 splice blocking morpholinos that scored negative in both primitive and definitive screens. In total, 51 of 57 morpholinos caused altered splicing, resulting in the same estimated FNR of $11 \%$. Furthermore, the knock down efficiencies were comparable to those that gave a hematopoietic defect (Supplementary Fig. 5).

\section{Classification of screen results}

Gene expression phenotypes observed in morpholino injected embryos, or morphants, were classified into one of three major categories: no change, decrease, or increase. Due to the range of decreased staining from subtle to complete absence of staining, we subdivided the decrease category into mild, intermediate, and strong (Fig. 2a-b, Supplementary Table 2). Any morphant showing changes in $\beta$-globin 33 or $c-m y b / r u n x l$ expression was considered a screen hit. Morphants with developmental abnormalities were listed separately (Supplementary Fig. 2a-b, Supplementary Table 2). As morphologically normal morphants with the strongest decrease or increase in blood formation represented genes that were likely to be specific to blood development, these 26 primitive and 47 definitive factors were selected for further characterization. 


\section{Chromatin factors regulate primitive blood development from the mesoderm}

Of the 26 morphants with altered primitive erythropoiesis, knockdown of 13 chromatin factors reduced $\beta$-globin e 3 expression and 13 factors increased $\beta$-globin e 3 expression at 16 ss. To confirm these phenotypes, we rescreened for $\beta$-globin e 3 expression. 16 of the 26 genes were verified, 6 from the reduced group and 10 from the increased group (Supplementary Table 3). Given that morpholinos can fail to inhibit their intended targets, splicing activity was confirmed by RT-PCR for the ten splice blocking morpholinos used, and a second, nonoverlapping morpholino was tested to verify the initial screen result for all factors. 15 of the 16 genes were validated in this manner and characterized further (Fig. 3ab, Supplementary Fig. 5). To show that the morpholinos did not just affect globin expression, one gene from each decrease category was reevaluated with a second erythroid marker, band3. The decrease in band 3 expression was consistent with the decrease in $\beta$ globin e 3 expression and was not rescued by $p 53$ loss (Fig. 3c), suggesting minimal, if any, morpholino toxicity. Overall, these additional tests provide further support for the validity of the screen results.

To examine early hematopoietic defects during the formation of mesodermal precursors and erythroid progenitors, we evaluated $s c l$ and gatal expression at 10-12ss (14hpf), respectively (Fig. 4) ${ }^{20}$. Of the 15 morphants tested, 12 showed changes in $\mathrm{scl}$ and gatal expression consistent with the changes in $\beta$-globin e 3 , suggesting that most of the factors categorized in the strong decrease or increase categories play a role in the formation of mesodermal precursors competent to make blood. Knockdown of one factor in particular, smarcal (or snf2l), strikingly abrogated expression of all three markers, implicating a requirement for this gene early in hematopoietic development (Supplementary Fig. 4a). The remaining three, chracl, actr $2 b$, and $h d a c 9 b$, had normal $s c l$ expression but reduced gatal and $\beta$-globin e 3 expression, indicating that these factors function to regulate the formation of erythroid progenitors from the mesoderm (Fig. 4). The majority of these factors represent previously unidentified regulators of erythroid development.

\section{Chromatin factors regulate the induction of HSPCs}

As in the primitive screen, we rescreened the 47 definitive genes ( 41 with a strong decrease and 6 with increase runxl/c-myb expression) and confirmed 31 genes: 26 morphants recapitulated the initial strong decrease in c-myb and runxl expression in the AGM at 36 hpf, and 5 were verified for increased $c-m y b$ and runxl expression (Supplementary Table 3). For morphants in the decrease category, expression of both markers was nearly abolished in the AGM. Morpholinos injected in a $553^{-/}$background did not rescue the loss of expression of AGM markers, indicating these phenotypes were not the result of morpholino toxicity (Fig. 3d). To verify the efficacy of the morpholinos, splicing activity was assessed and confirmed for all 21 splice blocking morpholinos used. 29 of the 31 genes were validated with a second, nonoverlapping morpholino and selected for further characterization (Fig. 3ab, Supplementary Fig. 5). Collectively, these data provide additional verification of our definitive screen data.

Previous work has shown that HSPCs emerge from the hemogenic endothelium of the dorsal aorta and that proper vessel development and establishment of artery identity is necessary 
for AGM stem cell induction ${ }^{23,24}$. The expression of vascular marker $f l k l$ and arterial identity marker ephrinB2 were analyzed for the 29 verified genes. The majority (20 of 29) showed normal $f l k 1$ and ephrinB2 expression. While these results do not directly demonstrate a cell autonomous mechanism, they do suggest that the chromatin factors identified in the strong decrease and increase categories function in the specification or maintenance of HSPCs from the hemogenic endothelium, as the tissues that arise most proximal to HSC specification were intact (Fig. 5). Both known stem cell regulators, such as hdacl and prdm16, and unknown factors, including brd8a, cbx6b, jmjdlc, and nap1l4a, were identified ${ }^{11,24}$.

The remaining ten chromatin factors were found to function at earlier stages of vessel specification based on the presence of vascular defects. Four of the factors, $h d a c 4 l, m b d 3 b$, phf $21 a$, and suv39hl, showed normal flk1 levels but reduced ephrinB2 expression, suggesting they function in the establishment of aorta identity upstream of HSPC formation . Finally, 5 morphants, mierl, jhdmlbb, jmjdlca, ing 4, and $r b b p 7$, lost both intersomitic flkl and arterial ephrinB2 expression, hence the loss of HSPCs in these morphants is likely due to the absence of hemogenic endothelium. Overall, our definitive screen uncovered chromatin regulators involved in the development of HSPCs from the AGM and during vascular development.

\section{Genes associated with mild to moderate knockdown phenotypes are important regulators of hematopoietic development}

In addition to chromatin factors with strong decrease or increase phenotypes, other factors with moderate to mild phenotypes also function in hematopoietic development. (Supplementary Table 3). We characterized seven genes from the primitive screen that showed only an intermediate reduction in $\beta$-globin e 3 expression upon reinjection. Of these seven morphants, six had normal $\mathrm{scl}$ but reduced gatal expression, suggesting that they likely function at the erythroid specification stage. Two of these factors, CHD4 and CBX8, associate with the FOG1/GATA1 transcriptional complex and the TIF1- $\gamma$ elongation complex, respectively; both complexes are key regulators of erythroid development ${ }^{25,26}$. Finally, kat 5 showed a decrease in $\beta$-globin e 3 without loss of scl and gatal expression, indicating it plays a role in erythroid cell differentiation. In comparison to the 15 genes with the strongest phenotypes, those with more mild phenotypes likely function at later stages of erythropoiesis after induction of the $\mathrm{scl}^{+}$mesoderm.

Similarly, for genes with moderate phenotypes from the definitive screen, we did not observe any defects in $f l k 1$ and ephrinB2 expression for 10 definitive morphants that were reconfirmed in the intermediate decrease category, suggesting these genes also regulate HSPC development. In summary, our screen has identified a large number of chromatin factors that contribute to different stages of primitive and definitive hematopoiesis.

\section{A chromatin factor interaction network for hematopoietic cell development}

Given that chromatin factors often function in multisubunit complexes, we sought to identify complexes important for hematopoietic development by mapping known protein interactions for the genes identified in our screen. We started by annotating the protein complexes for the 
44 validated genes from the primitive and definitive screens and included additional genes from the screen that were also present in these complexes ${ }^{27}$. Our results implicate multiple chromatin factor complexes required for developmental hematopoiesis: BAF/PBAF and ISWI (chromatin remodeling), SET1 (histone methylation - activation), HDAC/NuRD (histone deacetylation), NuA4/P300/CBP/HBO1 (histone acetylation), and PRC1/2 (histone methylation - repression) (Fig. 6, Supplementary Fig. 3).

To identify all possible chromatin complexes represented by our screen datasets, we generated a human protein-protein interaction map of the 425 chromatin factors screened and mapped the factors found in our screen (102 primitive and 116 definitive factors) onto the network. We identified the same complexes as we did in the previous analysis with the addition of a ubiquitination complex (Fig. 7, Supplementary Fig. 3).

As chromatin factors associated with the same complex likely share target binding sites, we analyzed 34 published ChIP-seq (chromatin immunoprecipitation followed by sequencing) datasets in K562 erythroleukemia cells of chromatin factors in our screen ${ }^{28}$. Two major complexes were found. The first group includes SIN3A, CHD4, HDAC1, TAF1, and JARID1C associated with the HDAC/NuRD complex, and the second group includes RNF2, SUZ12, CBX2, and CBX8 from the Polycomb complexes. We ranked triplet combinations of these factors together with all other groups of three factors based on the percent overlap of target genes. The HDAC/NuRD and PRC1/2 complex combinations predicted from our screen, including those which have been shown to interact biochemically, fell within the top $20 \%$ of all possible combinations of three factors (Supplementary Fig. 4a,c). After excluding a subset often factors that have large target gene lists (>8,000 target genes), which could skew the distribution, this filtered analysis resulted in the predicted interactions falling within the top 5\% of all combinations (Supplementary Fig. 4b,c). Both predicted interactions are significantly enriched in the upper tail of the distribution in the two analyses, therefore suggesting that our screen has identified chromatin factors that function in distinct complexes to regulate hematopoietic development.

\section{Genetic interaction of predicted chromatin complex subunits}

Based on complexes identified, we expected that chromatin factor subunits that scored positive in our screen would interact genetically. We tested the interaction by combinatorial knockdown of ISWI subunits required for primitive erythropoiesis, smarcal, chracl, and $r s f l b$ (Fig. 6) ${ }^{29,30}$. As described in the previous section, loss of these factors individually resulted in decreased gatal and $\beta$-globin e 3 expression. Knockdown of smarcal and $r s f l b$ also reduced $s c l$ expression. In knocking down all three factors at suboptimal doses, $s c l$ expression is retained while gatal and $\beta$-globin e 3 expression is highly reduced as in the single morphants (Fig. 8a). In contrast, knockdown of SWI/SNF components brgl, baf57, and baf170, which were not identified as primitive screen hits, did not result in primitive hematopoietic defects individually or in combination (Fig. 8b) ${ }^{31,32}$. These data suggest that the chromatin factors we identified from our screen interact genetically to regulate hematopoietic development. 


\section{Discussion}

Hematopoietic stem cells undergo proliferation and differentiation under the control of cellspecific transcription factors whose function is facilitated by chromatin factors. These factors establish an epigenetic landscape that controls self-renewal and provides lineage priming, driving differentiation. To better understand the epigenetic regulation of the hematopoiesis, we undertook the first reverse genetic approach to define the function of chromatin factors in the zebrafish. A library of zebrafish genes orthologous to 425 human chromatin factors were identified, containing canonical 'readers', 'writers', and 'erasers' of chromatin and other, less characterized, families.

In this study, we characterized a cohort of 15 chromatin factors that regulate primitive hematopoiesis and 29 that regulate definitive hematopoiesis, including both known and previously unidentified factors. Based on our validation work, the data suggest that these factors function at the level of erythroid and HSPC specification. Our analysis of several blood-specific markers at several distinct timepoints has been used to describe differentiation defects in many zebrafish blood mutants ${ }^{33,34}$. However, a delay in blood development would also readout as a decrease phenotype. As well, it is possible that an accelerated emergence of blood cells could confound our analysis except when it results in an overall increase in blood production. Regardless of whether blood development is selectively delayed or accelerated, our results ultimately show expression of the blood markers is altered, whether directly or indirectly affecting any number of pathways, such as metabolism, transcriptional elongation, and cell cycle regulation. Additional work will be required to determine the mechanism of action on hematopoiesis and whether these phenotypes are cell autonomous.

Disruption of both positive and negative regulators of chromatin frequently resulted in the same phenotype in our screen. For example, knockdown of $p 300$, which functions to acetylate histones, and $h d a c 6$, which deacetylates histones, each resulted in loss of $c$-my $b^{+}$ and runx $I^{+}$cells in the AGM. While they likely serve opposing roles in regulation of their respective target genes, their functions are both required for proper HSC specification.

These data are in concordance with proteomics data showing transcription factors, such as GATA1, recruiting both positive and negative regulators to activate and repress target genes, respectively ${ }^{35}$.

While members of the same chromatin family could compensate for each other, individual knockdown of many of these factors still resulted in a hematopoietic phenotype, suggesting nonredundant functions among related chromatin factors. Two factors that were identified from our screen, $p 300$ and crebbp, share similar functions but showed opposing phenotypes. p300 and CBP are homologous proteins that share a bromodomain and histone acetyltransferase domain ${ }^{36}$. Mouse knockouts of $\mathrm{p} 300$ and Cbp exhibit similar phenotypes ${ }^{37}$. Despite their overlapping roles, evidence of differential regulation has been accumulating. In HSCs, Cbp plays an important role in HSC self-renewal whereas p300 regulates HSC differentiation. Recent ChIP-seq results identified distinct binding sites between the two factors ${ }^{38,39}$. Consequently, the effect chromatin factors have in vivo cannot 
be predicted based solely on their domain function. Future in vivo studies will be important for our understanding of chromatin regulation and gene expression.

In a recent study investigating histone modifications on differentiating erythroid cells in mouse fetal liver, five histone marks were induced during this transition, $\mathrm{H} 3 \mathrm{~K} 4 \mathrm{me}$, H3K4me3, H3K9Ac, H4K16Ac, and H3K79me $2^{40}$. Consistent with these findings, our strongest primitive hits are composed of chromatin factors involved in methylation and acetylation of histones, including H3K4 methylation. Although similar work characterizing changes in histone marks during various stages of definitive HSC formation has not been done, we predict that they will include histone modifications such as methylation of H3K4, $\mathrm{H} 3 \mathrm{~K} 9$, and $\mathrm{H} 3 \mathrm{~K} 36$ based on the chromatin factors identified in our screen.

By examining our screen results, we identified relevant chromatin modifying complexes for blood development including BAF/PBAF, ISWI, HDAC/NuRD, NuA4/P300/CBP/HBO1, SET1, and PRC1/2 complexes. Hypotheses regarding the subunit composition of the chromatin factor complexes can be generated using our data set. One of the most striking results from our primitive screen was the knockdown of smarcal, which abrogates scl, gatal, and $\beta$-globin e 3 expression in the embryo. chracl and $r s f l b$, other components that form the ISWI complex, were also identified in the screen. In mammalian data, these factors form a complex with another family member, smarca5. Our data suggests that ISWI chromatin remodeling is important for primitive and definitive hematopoiesis and that the complex contains smarcal (not smarca5), chracl, and rsflb. As well, by comparing chromatin occupancy of complex members, we observed higher proportions of bound genes among factors predicted to be in the same complex. Taken together, these data suggest that our screen has identified chromatin factors that function in distinct complexes to regulate hematopoiesis.

Overall, we have identified a set of genes involved in the regulation of developmental hematopoiesis, including primitive erythropoiesis and definitive HSPC specification, and provide a resource for the identification and characterization of previously unidentified regulators. Studies focusing on the interactions between hematopoietic transcription factors and our chromatin factors will provide a more complete transcriptional network of gene regulation in blood development. In combination with other genetic and biochemical studies, our screen helps to unravel the epigenetic code that establishes the programs of gene expression for self-renewal and differentiation in hematopoietic cells.

\section{Supplementary Material}

Refer to Web version on PubMed Central for supplementary material.

\section{Acknowledgments}

We thank O. Tamplin, T.V. Bowman, P. Cahan, and C.K. Kaufman for helpful discussions. The work was supported by NIH NIDDK 5R01DK053298-15, NIH NHLBI 5R01HL048801-21, NIH NIDDK 5P30 DK49216-19, NIH NIDDK DK53298-15, NIH NIDDK R24 DK092760-02, HHMI (to L.I.Z.)), NIH NHLBI T32 HL066987-09 and NIH NIDDK 1F32DK089876-01 (to K.L.K)). L.I.Z. is a founder and stock holder of Fate, Inc. and Scholar Rock, and a scientific advisor for Stemgent. 


\section{References}

1. Li B, Carey M, Workman J. The role of chromatin during transcription. Cell. 2007; 128:707-726. [PubMed: 17320508]

2. Ho L, Crabtree G. Chromatin remodelling during development. Nature. 2010; 463:474-484. [PubMed: 20110991]

3. Cunliffe V. Histone deacetylase 1 is required to repress Notch target gene expression during zebrafish neurogenesis and to maintain the production of motoneurones in response to hedgehog signalling. Development (Cambridge, England). 2004; 131:2983-2995.

4. Gregg R, Willer G, Fadool J, Dowling J, Link B. Positional cloning of the young mutation identifies an essential role for the Brahma chromatin remodeling complex in mediating retinal cell differentiation. Proceedings of the National Academy of Sciences of the United States of America. 2003; 100:6535-6540. [PubMed: 12748389]

5. Miller C, Maves L, Kimmel C. moz regulates Hox expression and pharyngeal segmental identity in zebrafish. Development (Cambridge, England). 2004; 131:2443-2461.

6. Orkin S, Zon L. Hematopoiesis: an evolving paradigm for stem cell biology. Cell. 2008; 132:631644. [PubMed: 18295580]

7. Takihara Y. Role of Polycomb-group genes in sustaining activities of normal and malignant stem cells. International journal of hematology. 2008; 87:25-34. [PubMed: 18224410]

8. Yoshida T, et al. The role of the chromatin remodeler Mi-2beta in hematopoietic stem cell selfrenewal and multilineage differentiation. Genes Dev. 2008; 22:1174-1189. [PubMed: 18451107]

9. Krivtsov A, Armstrong S. MLL translocations, histone modifications and leukaemia stem-cell development. Nature reviews Cancer. 2007; 7:823-833. [PubMed: 17957188]

10. Duhoux FP, et al. PRDM16 (1p36) translocations define a distinct entity of myeloid malignancies with poor prognosis but may also occur in lymphoid malignancies. Br J Haematol. 2012; 156:7688. [PubMed: 22050763]

11. Chuikov S, Levi B, Smith M, Morrison S. Prdm16 promotes stem cell maintenance in multiple tissues, partly by regulating oxidative stress. Nature cell biology. 2010; 12

12. Shipra A, Chetan K, Rao MR. CREMOFAC--a database of chromatin remodeling factors. Bioinformatics. 2006; 22:2940-2944. [PubMed: 17021159]

13. Schultz J, Milpetz F, Bork P, Ponting CP. SMART, a simple modular architecture research tool: identification of signaling domains. Proc Natl Acad Sci U S A. 1998; 95:5857-5864. [PubMed: 9600884]

14. Marchler-Bauer A, et al. CDD: a Conserved Domain Database for the functional annotation of proteins. Nucleic Acids Res. 2011; 39:D225-229. [PubMed: 21109532]

15. Punta M, et al. The Pfam protein families database. Nucleic Acids Res. 2012; 40:D290-301. [PubMed: 22127870]

16. Gendler K, Paulsen T, Napoli C. ChromDB: the chromatin database. Nucleic Acids Res. 2008; 36:D298-302. [PubMed: 17942414]

17. Kimmel C, Ballard W, Kimmel S, Ullmann B, Schilling T. Stages of embryonic development of the zebrafish. Developmental dynamics: an official publication of the American Association of Anatomists. 1995; 203:253-310. [PubMed: 8589427]

18. Brownlie A, et al. Characterization of embryonic globin genes of the zebrafish. Developmental biology. 2003; 255:48-61. [PubMed: 12618133]

19. North T, et al. Prostaglandin E2 regulates vertebrate haematopoietic stem cell homeostasis. Nature. 2007; 447:1007-1011. [PubMed: 17581586]

20. Liao E, et al. SCL/Tal-1 transcription factor acts downstream of cloche to specify hematopoietic and vascular progenitors in zebrafish. Genes \& development. 1998; 12:621-626. [PubMed: 9499398]

21. Muralidhar SA, Ramakrishnan V, Kalra IS, Li W, Pace BS. Histone deacetylase 9 activates gamma-globin gene expression in primary erythroid cells. J Biol Chem. 2011; 286:2343-2353. [PubMed: 21078662] 
22. Wang J, et al. ASH2L: alternative splicing and downregulation during induced megakaryo cytic differentiation of multipotential leukemia cell lines. Journal of molecular medicine (Berlin, Germany). 2001; 79:399-405.

23. Kissa K, Herbomel P. Blood stem cells emerge from aortic endothelium by a novel type of cell transition. Nature. 2010; 464:112-115. [PubMed: 20154732]

24. Burns C, et al. A genetic screen in zebrafish defines a hierarchical network of pathways required for hematopoietic stem cell emergence. Blood. 2009; 113:5776-5782. [PubMed: 19332767]

25. Hong W, et al. FOG-1 recruits the NuRD repressor complex to mediate transcriptional repression by GATA-1. The EMBO journal. 2005; 24:2367-2445. [PubMed: 15920470]

26. Bai X, et al. TIF1gamma controls erythroid cell fate by regulating transcription elongation. Cell. 2010; 142:133-143. [PubMed: 20603019]

27. Apweiler R JMM, et al. Reorganizing the protein space at the Universal Protein Resource (UniProt). Nucleic Acids Res. 2012; 40:D71-75. [PubMed: 22102590]

28. Peter BB. A User' Guide to the Encyclopedia of DNA Elements (ENCODE). PLoS biology. 2011; 9

29. LeRoy G, Orphanides G, Lane WS, Reinberg D. Requirement of RSF and FACT for transcription of chromatin templates in vitro. Science. 1998; 282:1900-1904. [PubMed: 9836642]

30. Varga-Weisz P, Becker P. Chromatin-remodeling factors: machines that regulate? Current opinion in cell biology. 1998; 10:346-399. [PubMed: 9640535]

31. Wang W, et al. Architectural DNA binding by a high-mobility-group/kinesin-like subunit in mammalian SWI/SNF-related complexes. Proceedings of the National Academy of Sciences of the United States of America. 1998; 95:492-498. [PubMed: 9435219]

32. Wang W, et al. Diversity and specialization of mammalian SWI/SNF complexes. Genes \& development. 1996; 10:2117-2130. [PubMed: 8804307]

33. Ransom D, et al. Characterization of zebrafish mutants with defects in embryonic hematopoiesis. Development (Cambridge, England). 1996; 123:311-319.

34. Thompson M, et al. The cloche and spadetail genes differentially affect hematopoiesis and vasculogenesis. Developmental biology. 1998; 197:248-269. [PubMed: 9630750]

35. Rodriguez $\mathrm{P}$, et al. GATA-1 forms distinct activating and repressive complexes in erythroid cells. The EMBO journal. 2005; 24:2354-2420. [PubMed: 15920471]

36. Ogryzko V, Schiltz R, Russanova V, Howard B, Nakatani Y. The transcriptional coactivators p300 and CBP are histone acetyltransferases. Cell. 1996; 87:953-962. [PubMed: 8945521]

37. Yao T, et al. Gene dosage-dependent embryonic development and proliferation defects in mice lacking the transcriptional integrator p300. Cell. 1998; 93:361-433. [PubMed: 9590171]

38. Rebel V, et al. Distinct roles for CREB-binding protein and p300 in hematopoietic stem cell selfrenewal. Proceedings of the National Academy of Sciences of the United States of America. 2002; 99:14789-14883. [PubMed: 12397173]

39. Ramos Y, et al. Genome-wide assessment of differential roles for $\mathrm{p} 300$ and CBP in transcription regulation. Nucleic acids research. 2010; 38:5396-5804. [PubMed: 20435671]

40. Wong $\mathrm{P}$, et al. Gene induction and repression during terminal erythropoiesis are mediated by distinct epigenetic changes. Blood. 2011; 118:38.

41. Lawrence C, Adatto I, Best J, James A, Maloney K. Generation time of zebrafish (Danio rerio) and medakas (Oryzias latipes) housed in the same aquaculture facility. Lab Anim (NY). 2012; 41:158165. [PubMed: 22614091]

42. Berghmans, Sp, et al. tp53 mutant zebrafish develop malignant peripheral nerve sheath tumors. Proceedings of the National Academy of Sciences of the United States of America. 2005; 102:407-412. [PubMed: 15630097]

43. Kimmel CB, Ballard WW, Kimmel SR, Ullmann B, Schilling TF. Stages of embryonic development of the zebrafish. Dev Dyn. 1995; 203:253-310. [PubMed: 8589427]

44. Shimoda N, Yamakoshi K, Miyake A, Takeda H. Identification of a gene required for de novo DNA methylation of the zebrafish no tail gene. Developmental dynamics: an official publication of the American Association of Anatomists. 2005; 233:1509-1525. [PubMed: 15937923] 
45. Thisse C, Thisse B. High-resolution in situ hybridization to whole-mount zebrafish embryos. Nature protocols. 2008; 3:59-128. [PubMed: 18193022]

46. Turner B, et al. iRefWeb: interactive analysis of consolidated protein interaction data and their supporting evidence. Database (Oxford). 2010; 2010:baq023. [PubMed: 20940177]

47. Lopes CT, et al. Cytoscape Web: an interactive web-based network browser. Bioinformatics. 2010; 26:2347-2348. [PubMed: 20656902]

48. McLean C, et al. GREAT improves functional interpretation of cis-regulatory regions. Nature biotechnology. 2010; 28:495-501. 


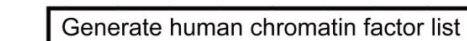
from public databases $(n=425)$

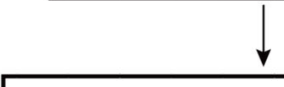

Identify zebrafish orthologs by BLAST $(n=488)$

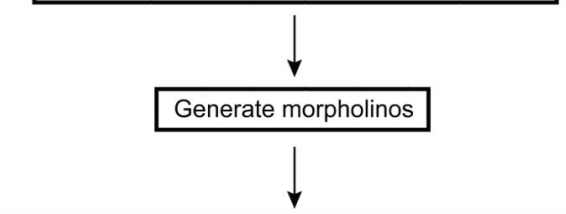

Inject morpholinos into single-cell zebrafish embryos

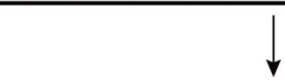

Collect embryos at $17 \mathrm{hpf}$ and $36 \mathrm{hpf}$ for WISH

Score embryos for changes in globin or c-myb/runx1 expression

b

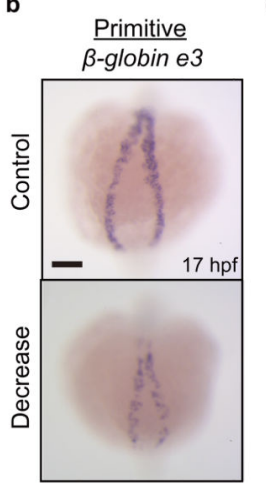

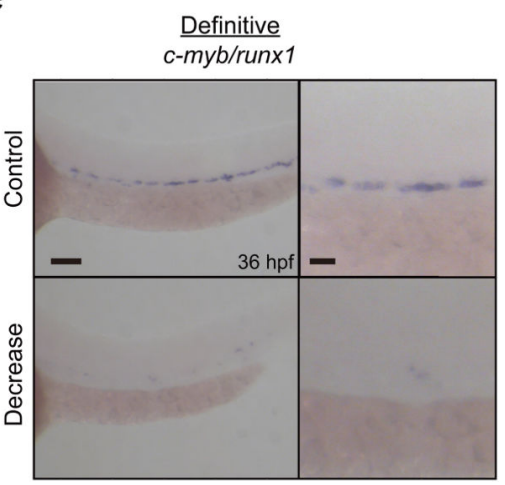

Figure 1.

Screen design for chromatin regulators of developmental hematopoiesis. (a) Schematic illustration of screening procedure. Knockdown of 488 zebrafish orthologs of 425 human chromatin factors was achieved using morpholinos. Embryos were subsequently collected at $17 \mathrm{hpf}$ and $36 \mathrm{hpf}$ to analyze changes in $\beta$-globin e 3 expression in primitive erythroid cells and $c-m y b$ and runxl expression in definitive HSPCs, respectively, by WISH. (b) Example of primitive screen phenotype observed for reduced $\beta$-globin e 3 expression at $17 \mathrm{hpf}$. (c) Example of definitive screen phenotype observed for reduced c-myb and runxl expression at $36 \mathrm{hpf}$. Scale bars: $100 \mu \mathrm{m}$ for low magnification and $25 \mu \mathrm{m}$ for high magnification. 

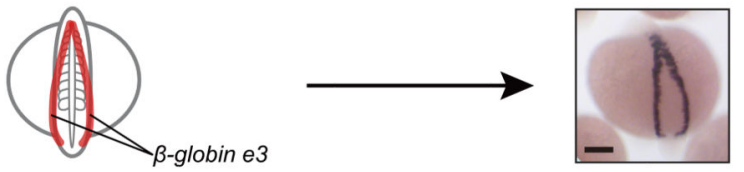

$16 \mathrm{ss}$ (17 hpf)

WT

(posterior tail view)

\begin{tabular}{|c|c|c|c|c|c|}
\hline \multicolumn{5}{|c|}{ Primitive screen results $(\mathrm{n}=488)$} & Decrease $(\mathrm{n}=93)$ \\
\hline No change $(\mathrm{n}=288)$ & & Increase $(\mathrm{n}=9)$ \\
\hline & & & & \\
\hline
\end{tabular}

b
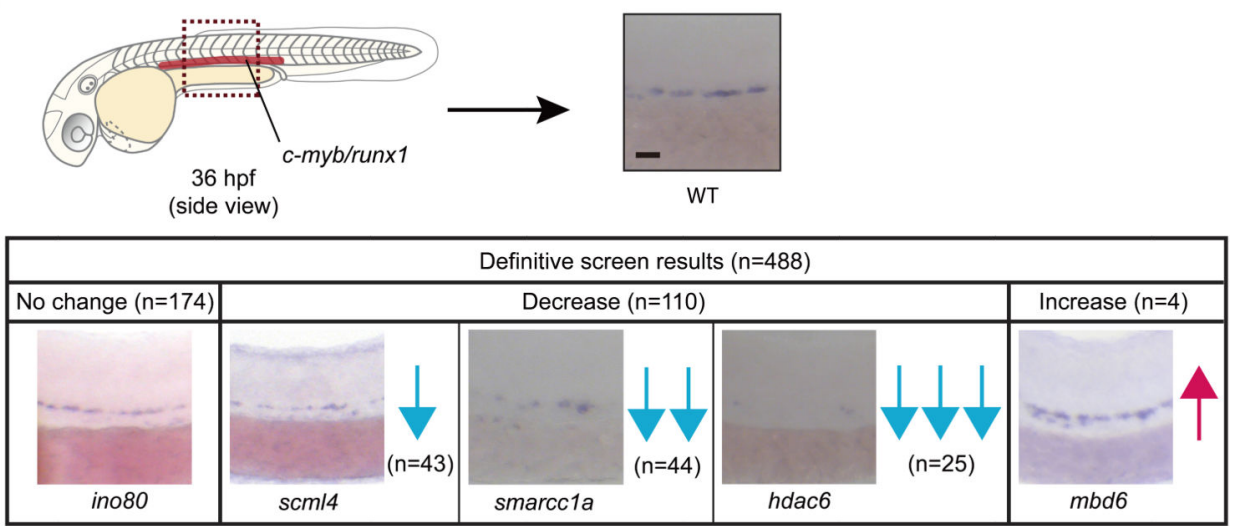

Figure 2.

Classification of screen results. (a) Summary of primitive screen WISH results. Developing $\beta$-globin $^{+}$erythroid cells are found as two bilateral stripes in the posterior of the embryo, as shown by the red highlights in the schematic of a 16 ss embryo. Knockdown of the different chromatin factors resulted in no change, decrease, or increase of $\beta$-globin e 3 expression. (b) Summary of definitive screen WISH results. Induction of $c-m y b^{+}$and runxl $I^{+}$ HSPCs occurs in the AGM region highlighted in red in the schematic of a $36 \mathrm{hpf}$ embryo. Knockdown of the different chromatin factors screened resulted in no change, decrease, or increase of $c-m y b$ and runxl expression. Representative WISH results are shown for each phenotypic category with additional categories continued in Supplementary Fig. 2. " $n$ " is the number of chromatin factors with the indicated phenotype. Blue downward arrows represent reduced marker expression and magenta upward arrows represent increased marker expression. One arrow indicates a mild change, two arrows an intermediate change, and three arrows a strong change. Scale bars: $100 \mu \mathrm{m}$ 

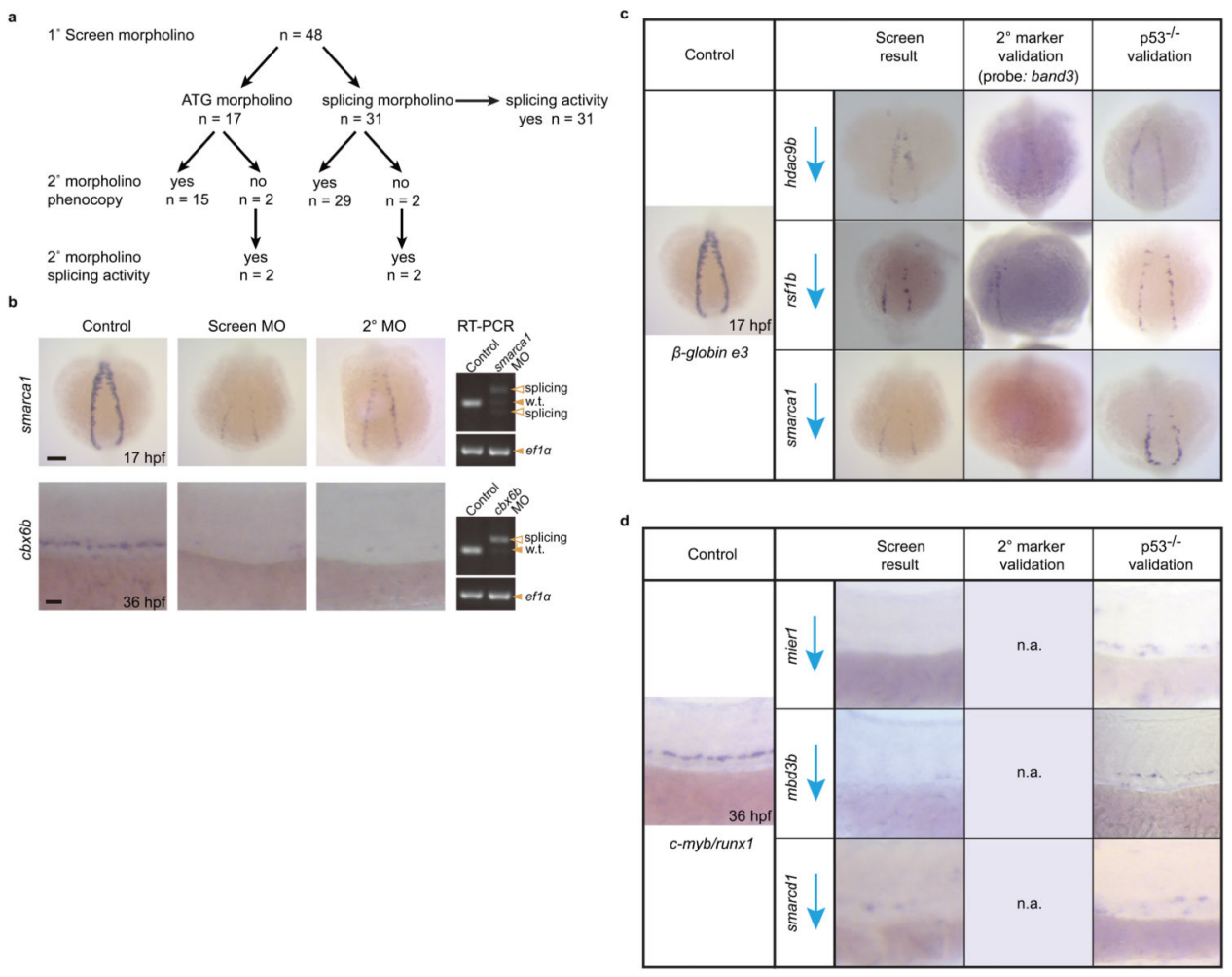

Figure 3.

Morpholino efficacy and secondary verification of screen phenotypes. (a) Flow chart of screening morpholino validation procedure. 44 of the 48 genes identified from the screen were verified with a second, nonoverlapping morpholino. The remaining 4 were recategorized. Splicing activity for splice blocking morpholinos were assayed and confirmed by RT-PCR for the 31 primary screen morpholinos and the 4 secondary morpholinos that did not phenocopy the primary screen results. $n=$ is the number of morpholinos under each category. (b) Example of primitive and definitive screen hits that were verified with a secondary morpholino and show splicing activity by RT-PCR. efl $a$ was used as the control gene. Arrowheads mark the presence of a PCR band. Filled in arrowheads indicate wild-type bands and empty arrowheads indicate spliced bands. w.t stands for wild-type. (c) A subset of 3 primitive genes that resulted in decreased globin expression when knocked down were selected, one from each decrease category, to test for expression of a second erythroid marker, band3.(d) A subset of 3 definitive genes that resulted in decreased $c$-myb and runx 1 expression when knocked down were selected, one from each category shown in Fig. 5. Given that two probes were used during the screen and expression of both genes were nearly abolished in the morphants, only the $p 53^{-/-}$rescue experiment was performed. n.a. stands for not applicable. Scale bars: $100 \mu \mathrm{m}$ for low magnification and $25 \mu \mathrm{m}$ for high magnification. 


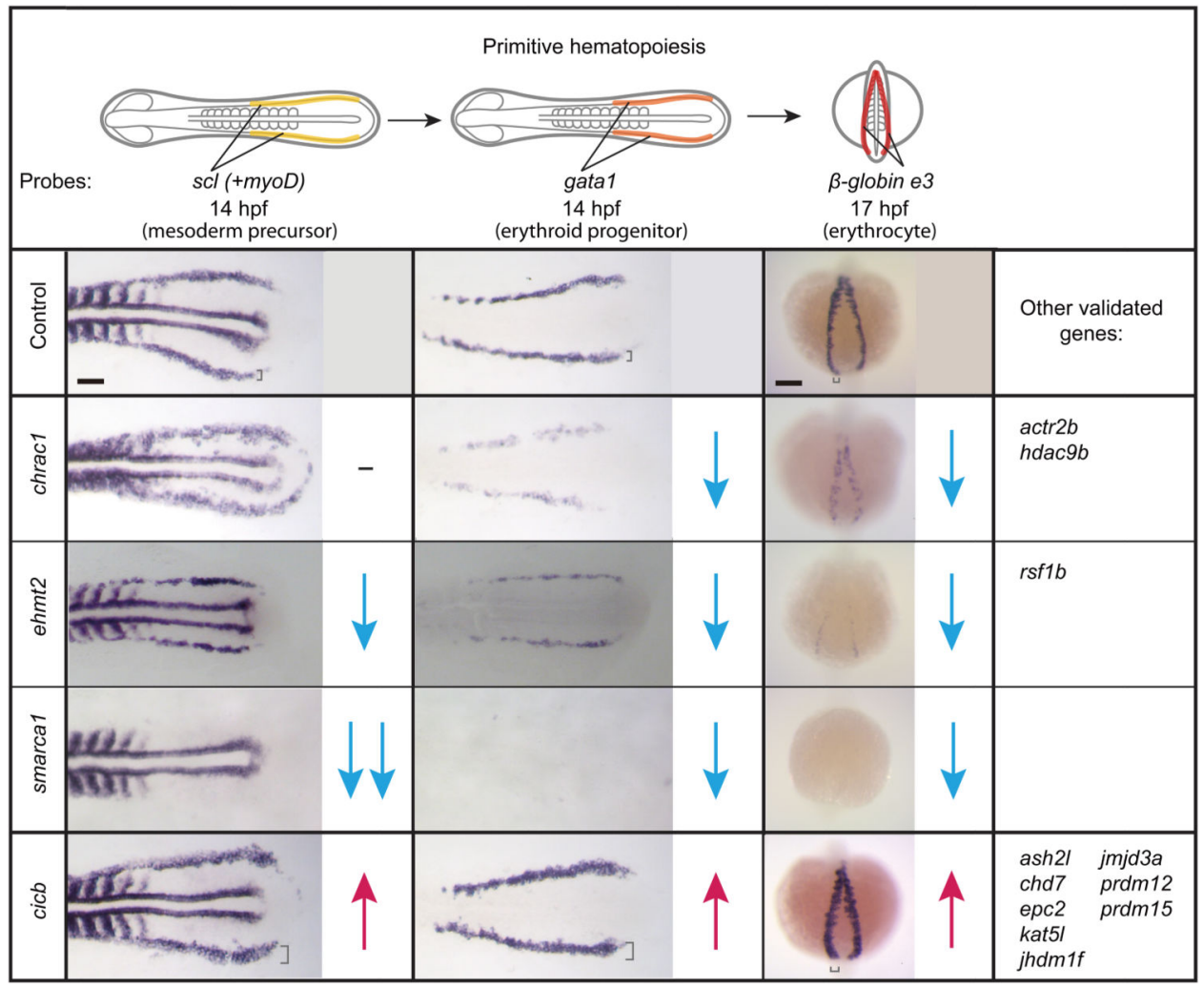

Figure 4.

Chromatin factors regulate distinct steps of primitive erythroid development. 15 primitive genes were screened for changes in $\mathrm{scl}$ expression marking hematopoietic mesodermal precursors (yellow highlight) and gatal expression marking erythroid progenitor formation (orange highlight) illustrated in the top panel (flatmount view). $\beta$-globin e3 staining was repeated to verify primary screen results (posterior view). myoD probe for labeling somites was included as a staging marker. Blue downward arrow represents reduced expression. Magenta upward arrow represents increased expression. A hyphen indicates no change in gene expression. Square brackets indicate the thickness of the stripes. Scale bar: $100 \mu \mathrm{m}$ 


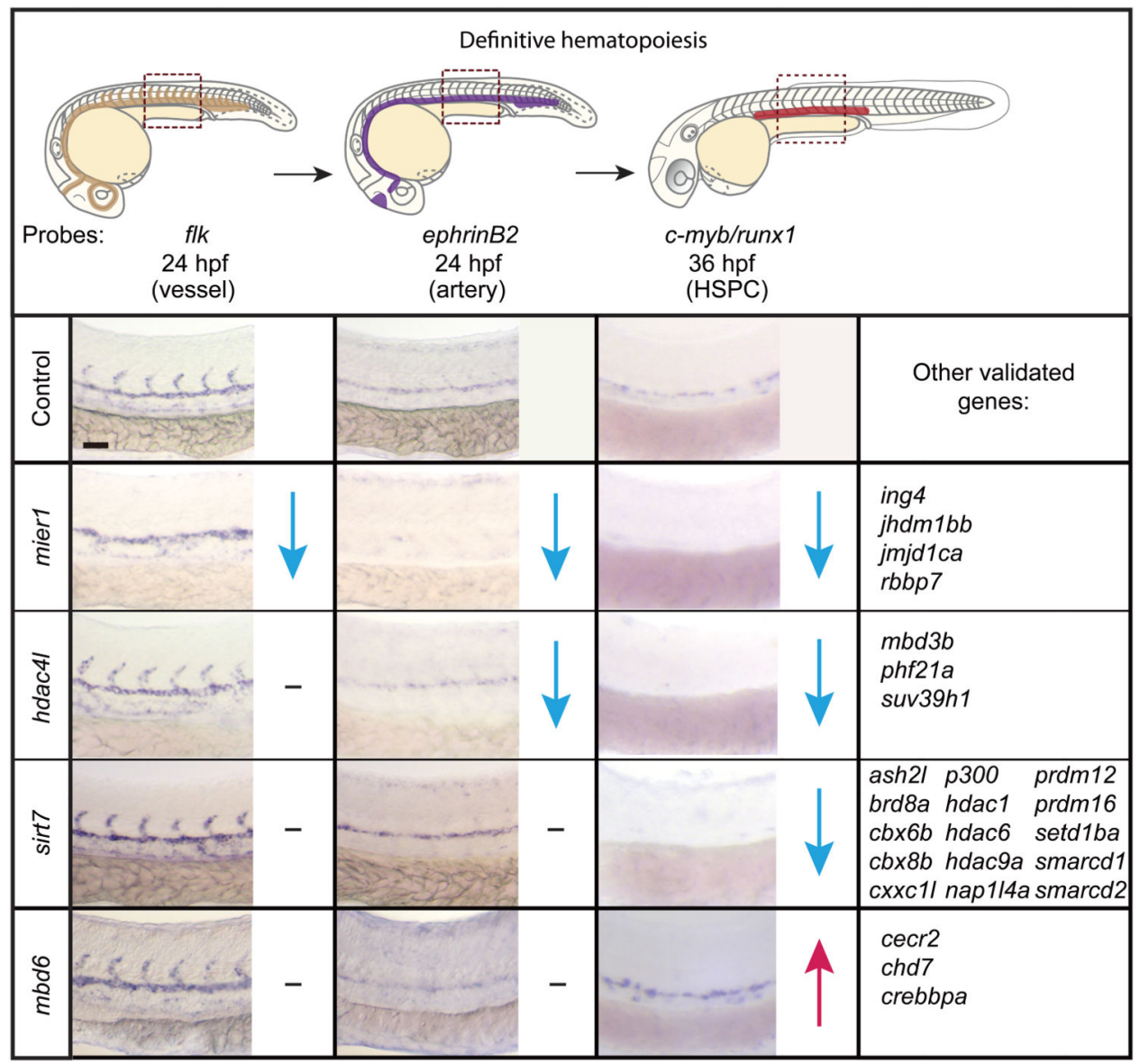

Figure 5.

Chromatin factors regulate distinct steps of definitive HSPC development. 29 definitive genes were screened for changes in $f l k l$ expression marking the vessels (tan highlight) and ephrinB2 expression marking the artery endothelium (purple highlight) illustrated in the top panel. c-myb and runxl staining was repeated to verify primary screen results. Blue downward arrow represents reduced expression. Magenta upward arrow represents increased expression. A hyphen indicates no change in gene expression. Scale bars: $100 \mu \mathrm{m}$ for low magnification and $25 \mu \mathrm{m}$ for high magnification. 


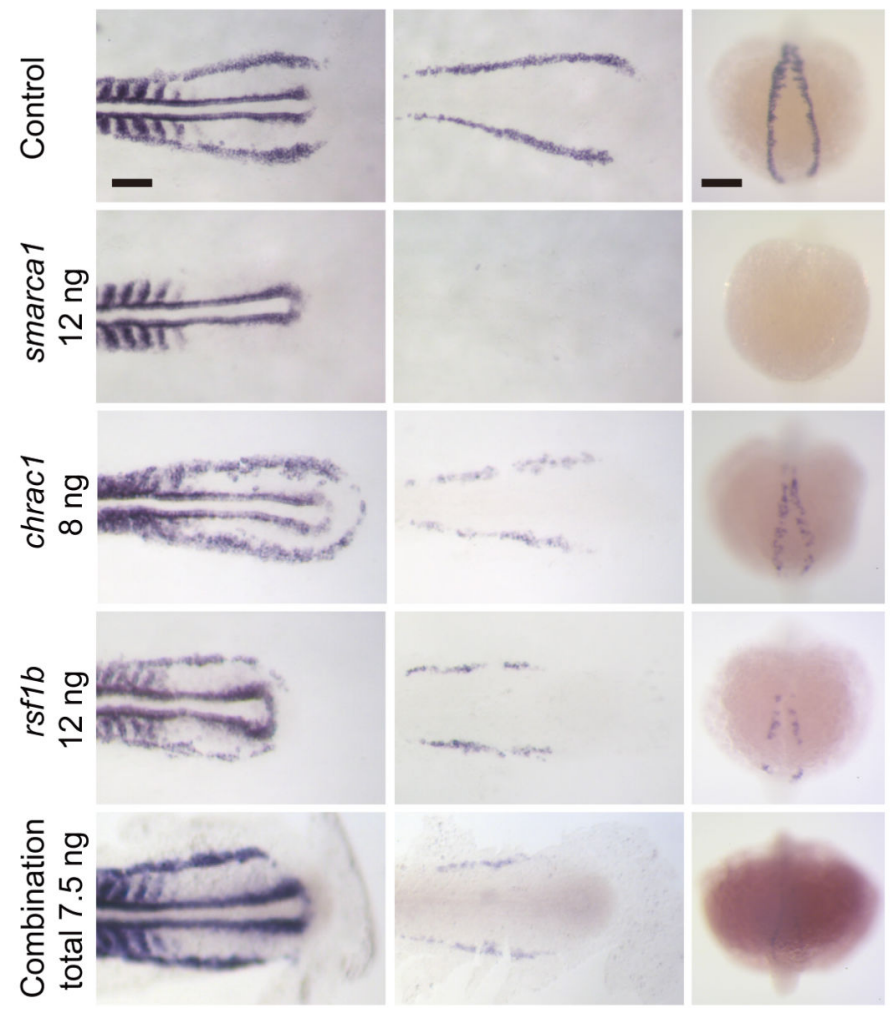

b

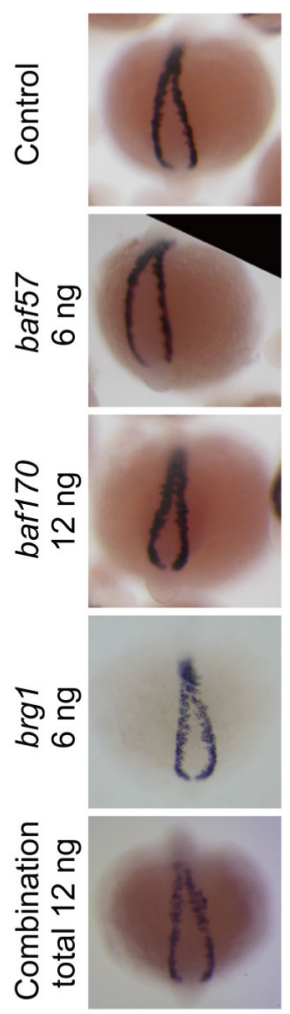

Figure 6.

Identification of chromatin modifying complexes using protein interaction data for the 44 validated primitive and definitive genes. Protein complexes associated with the 44 chromatin factors from the decrease (strong) and increase categories were identified, and additional genes from the screen present in these complexes were included (Uniprot).

Results for SWI/SNF, ISWI, SET1, and CBP/P300 are illustrated with additional complexes shown in Supplementary Figure 3. Filled in circles represent chromatin factors that were identified as screen hits with their respective phenotypic classification denoted by different colors. Dotted lines indicate alternative complex associations. 


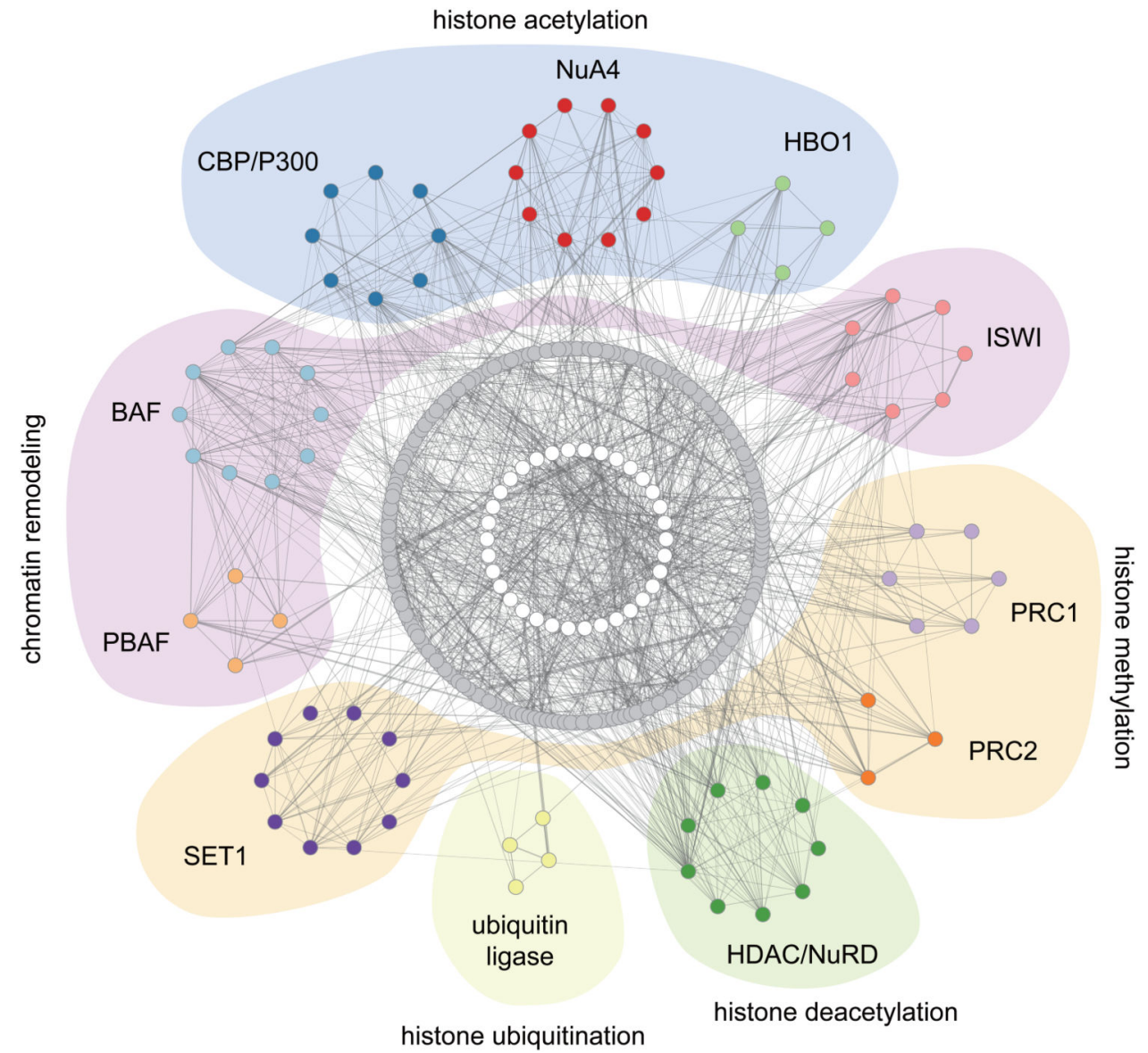

Figure 7.

A protein-protein interaction network for the 425 human chromatin factors screened. Screen results for 102 primitive and 116 definitive genes were mapped onto the network, and highly interconnected modules containing factors identified from the screens were isolated, representing the chromatin complexes important for hematopoietic development ${ }^{39}$. Detailed maps of the modules are in Supplementary Figure 5. Each module is assigned a unique color, and those that share the same chromatin function are highlighted in the same background color. Grey circles represent chromatin factors not associated with the isolated complexes, and white circles mark additional factors that were added to increase the connectivity of the network. 

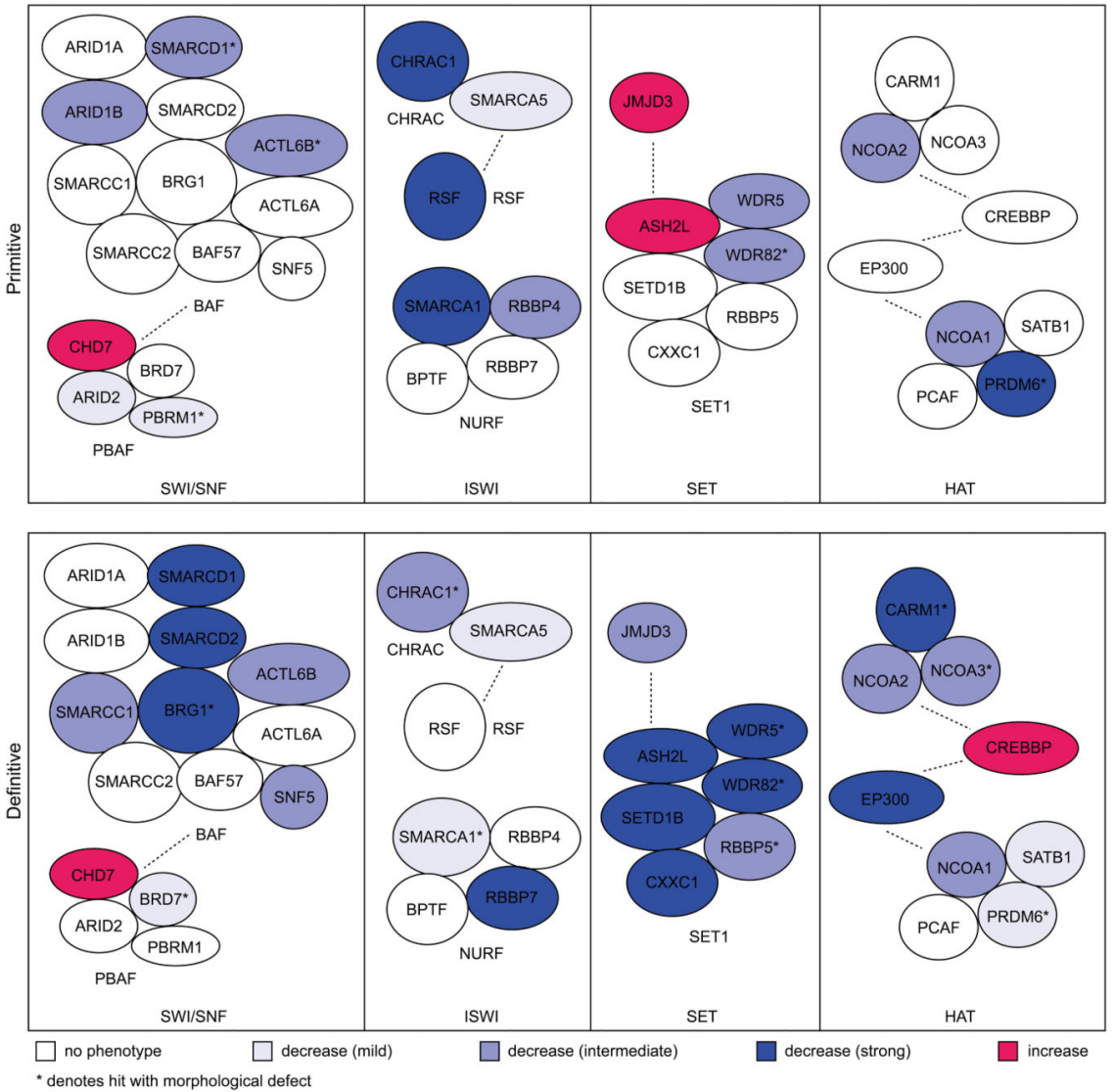

Figure 8.

Genetic interaction of ISWI chromatin factors by combinatorial knockdown. (a) gatal and $\beta$-globin e3 expression levels were examined by injecting single and combined suboptimal doses of morpholinos against smarcal, chracl, and $r s f l b$. Combined dose of $7.5 \mathrm{ng}$ includes $4 \mathrm{ng}$ of smarcal, $0.8 \mathrm{ng}$ of chracl, and $2.7 \mathrm{ng}$ of rsflb.(b) Knockdown of SWI/SNF chromatin factors baf57, bafl70, and brgl, which were not identified as hits from the screen, were tested for $\beta$-globin e 3 expression either individually or in combination. Combined dose of $12 \mathrm{ng}$ includes $4 \mathrm{ng}$ of each of the three morpholinos. Morpholino doses are indicated as nanograms (ng). Scale bars: $100 \mu \mathrm{m}$ 